(c) American Dairy Science Association, 2007.

\title{
Genetic Impacts of Using Female-Sorted Semen in Commercial and Nucleus Herds
}

\author{
G. Abdel-Azim ${ }^{1}$ and S. Schnell \\ Genex Cooperative Inc., Cooperative Resources International, 100 MBC Drive, Shawano, WI 54166
}

\begin{abstract}
This study was designed to investigate the genetic effects of using sorted semen in a dairy cattle population. Progress was monitored in elite and commercial animals over $20 \mathrm{yr}$ of selection. To study the genetic impact of using sorted semen in commercial herds, a scenario was evaluated in which female-sorted semen was available to commercial herds. Second, to study the genetic impact of using sorted semen in nucleus herds, scenarios were simulated in which female-sorted semen was used only in a centralized nucleus herd, in which multiple ovulation and embryo transfer (MOET) took place. Because of the additional advantage of marker-assisted selection when sorted semen was used in nucleus herds, a second scenario was simulated in which both sorted semen and marker-assisted selection were implemented. In the scenario in which femalesorted semen was used in commercial herds, a large genetic advantage was observed early in commercial cows. The average superiority in first-lactation cows exceeded $30 \%$ in yr 11, relative to a base scheme with regular semen, but continued to decrease until it reached $9 \%$ in yr 20 . The increased selection intensity in commercial cows contributed to the genetic merit of future cows (cow-to-cow contribution), but the contribution of the nucleus grew over time and gradually marginalized the cow-to-cow contribution. The genetic advantage of gender control in MOET schemes was minimal except when marker-assisted selection was also available. Two factors that affected the contribution of marker-assisted selection were studied: 1) within- vs. across-family selection of donors, and 2) the number of loci in the quantitative trait locus component. Schemes that selected donors regardless of their family structure were superior, and the quantitative trait locus component with more loci increased the effectiveness of sorted semen. Finally, we studied a reduced MOET scheme in which the number of harvested females was reduced from 42 to $25 / \mathrm{yr}$. The reduced scheme in combination
\end{abstract}

Received March 22, 2006.

Accepted October 19, 2006.

${ }^{1}$ Corresponding author: gamal@crinet.com with female-sorted semen was not found to be genetically inferior to the large scheme in combination with regular semen.

Key words: dairy cattle breeding, sorted semen, marker-assisted selection, multiple ovulation and embryo transfer

\section{INTRODUCTION}

Genetic effects of gender control have not been given much attention in the past because the ability to control the gender of the young produced was merely an improbable theory. Lush (1945) theorized that such control would require a treatment to either destroy the fertilized eggs of one sex and leave the other unharmed or separate the 2 kinds of spermatozoa, perhaps by killing 1 of the 2 kinds. Lush (1945) speculated: "The improbability of there being such a treatment, or finding it even if it does exist, becomes evident when it is considered that in the farm animals the two kinds of spermatozoa would be exactly alike in well over 90 per cent of the material they contain" (p. 408). Ironically, although the difference between $\mathrm{X}$ - and Y-bearing spermatozoa is known to be well under $10 \%$, it is now possible to separate them.

Genetic effects of some forms of gender control in specialized schemes of dairy cattle breeding have been studied. Embryo sexing within closed mixed multiple ovulation and embryo transfer (MOET) schemes was evaluated by Colleau (1991) and was found to improve adult MOET schemes and make them an efficient alternative to juvenile schemes without embryo sexing. Colleau (1985) reported a 15\% inferiority of MOET adult schemes relative to juvenile schemes, even after optimizing nucleus size for a given overall number of embryos transferred and after taking into account the Bulmer effect. Kinghorn et al. (1991) and Weigel (2004) further suggested the use of within-family selection (WFS) of full-sibs of one sex in juvenile MOET schemes, using auxiliary information such as indicator traits or marker loci.

In general schemes of dairy cattle breeding, Van Vleck (1981) concluded that the rate of genetic progress could increase by $15 \%$ if sorted semen (SS) were widely available. However, Baker et al. (1990) predicted much 
Table 1. Breeding systems simulated and comparisons made among them to study genetic impacts of using female-sorted semen ( 9 SS)

\begin{tabular}{|c|c|c|}
\hline Comparison & Base breeding system & Use of $\mathrm{SS}^{1}$ \\
\hline 2 & Mixed ET ${ }^{2}$ scheme & Base system $2+\uparrow \mathrm{SS}$ exclusively available to a centralized nucleus herd \\
\hline 3 & Mixed ET scheme + MAS $^{3}$ (1 locus) & Base system $3+q \mathrm{SS}$ exclusively available to a centralized nucleus herd \\
\hline 5 & Mixed ET scheme + MAS (1 locus) + WFS $^{4}$ & Base system $5+q$ SS exclusively available to a centralized nucleus herd \\
\hline 6 & Mixed ET scheme + MAS (4 loci) + WFS & Base system $6+q \mathrm{SS}$ exclusively available to a centralized nucleus herd \\
\hline 7 & Mixed ET ${ }^{2}$ scheme + MAS (4 loci) & $\begin{array}{l}\text { Small }{ }^{5} \text { mixed ET scheme + MAS (4 loci) }+ \text { \&S exclusively available to } \\
\text { a centralized nucleus herd }\end{array}$ \\
\hline
\end{tabular}

${ }^{1} \mathrm{X}$-bearing sperm cells (85\% expected female calves).

${ }^{2}$ Embryo transfer (ET) based on 42 donors; contributes $50 \%$ of young bulls.

${ }^{3}$ Marker-assisted selection (MAS), based on 1 and 4 loci.

${ }^{4}$ Within-family selection (WFS), selecting the best female embryo from each full-sib family.

${ }^{5}$ Embryo transfer based on 25 donors.

less progress by altering the sex ratio of the offspring of elite sires.

The objective of the current study was to quantify the impact of using SS on the genetic progress of a livestock population. Stochastic computer simulation was used with parameters proportional to the US Holstein population. In addition, the study investigated the impact of SS in combination with 2 other biotechnological factors, marker-assisted selection (MAS) and MOET.

\section{MATERIALS AND METHODS}

The current simulation used population structure and selection practices similar to those of the US Holstein population. Although the population numbers used in the simulation were smaller than true numbers because of the stochastic nature of the simulation, the simulated population structure was a replica of the true structure. Population structure and selection practices should mainly drive the results in this study. Details of the stochastic simulation model can be found in AbdelAzim and Freeman (2002). Progress was monitored in elite and commercial herds over $20 \mathrm{yr}$ of selection. First, to study the genetic impact of using SS in commercial herds, a scenario was simulated in which female-SS ( $q$ SS) was available to commercial herds. Second, to study the genetic impact of using SS in nucleus herds, scenarios were simulated in which 9 SS was used only in a centralized nucleus herd in which MOET took place. Finally, because of the additional advantage of MAS when SS is used in nucleus herds (Weigel, 2004), use of SS was simulated in scenarios that used MAS.

\section{Description of the Breeding Systems Studied}

Table 1 lists the breeding systems studied and the relevant comparisons made among them. In compari- son 1 , a conventional 2-stage selection system was simulated and compared with a similar system in which o SS was widely available to commercial herds. In this scenario, producers used + SS on top females to produce future herd replacements. With $+\mathrm{SS}$, fewer females were needed to produce herd replacements, the best $38 \%$ vs. the best $66 \%$ in the case of regular semen (RS). Commercial herds were expected to benefit the most from such use of i SS because of the increased selection intensity of replacement heifers. However, the trends and magnitudes of genetic benefits were quantified for both commercial and elite animals over a 20 -yr period.

Second, in comparison 2 (Table 1), a centralized nucleus herd that contributed $50 \%$ of the young bulls (YB) tested annually was simulated. The nucleus herd used MOET and was based on 42 donors, harvested annually. The nucleus herd was simulated as an addition to a progeny-testing scheme that provided the other $50 \%$ of the YB tested annually. This constituted a base mixed embryo transfer (ET) scheme in which genetic progress was essentially boosted because of its reproductive advantage. To study the effect of SS in this scheme, o SS was made available to nucleus donor females but not to commercial herds, as in comparison 1. Seventeen female embryos and 3 male embryos resulted from each donor per year vs. 10 female and 10 male embryos in the case of RS. This increased the selection intensity of donor females in the nucleus herd. However, this increase in intensity was not expected to be effective because of the larger full-sib families from which future donors were selected. This led to the simulation of other scenarios in which MAS was made available in the mixed ET scheme. The availability of MAS made differentiation among full-sib donors possible. Four base systems were simulated in which both MOET and MAS took place (comparisons $3,4,5$, and 6 in Table 1). In comparisons 3 and 4, individual donors were selected annually based on estimated QTL and polygenic compo- 
Table 2. Parameter values used in the simulation

\begin{tabular}{ll}
\hline Parameter & Value \\
\hline Ratio of X-bearing sperm cells in female-sorted semen & $85 \%$ \\
Portion of genetic variance associated with known QTL & 15 and $3.75 \%$ \\
Average number of daughters per YB $^{1}$ & 60 \\
Number of YB/yr & 21 \\
Number of AS $/$ yr & 10 \\
Number of harvested embryos/donor per yr & 20 \\
Average number of milking cows/yr & 10,000 \\
Ratio of parities 1 to 5 & $0.33,0.26,0.19,0.14$, and 0.09, respectively \\
Culling ratios for parities 1 to 5 & $0.22,0.26,0.29,0.34$, and 1.0, respectively \\
\hline 1'Young bull. & \\
${ }^{2}$ Active sires. &
\end{tabular}

nents, regardless of the number selected from the same full-sib family. Therefore, the best 42 donors were selected annually from 420 female embryos in the case of RS and from 714 female embryos in the case of SS. Comparisons 5 and 6 were similar to 3 and 4 except that WFS was forced on the selection of donors, in which only one donor was selected from each full-sib family. Without MAS, any WFS was performed at random; therefore, scenarios 5 and 6 were designed to increase the effectiveness of MAS in the mixed ET scheme.

In the mixed ET system, o SS increased the number of female embryos produced. This increase was needed to capture all marker diversity, which should further increase the effectiveness of MAS when donors are selected. With only one QTL, diversity was limited to 3 different genotypes, but with 4 QTL diversity was $3^{4}=$ 81 different genotypes. Hence, availability of SS was expected to be more effective in combination with MAS in mixed ET schemes. This led us to compare 2 MAS schemes: In the first scheme, only one QTL was simulated (comparisons 3 and 5), and in the second scheme, 4 QTL were simulated (comparisons 4 and 6). The same portion of the additive genetic variation $(15 \%)$ was attributed to the QTL component; that is, in the case of one QTL, all the $15 \%$ was attributed to the QTL, but in the case of 4 QTL, 3.75\% was attributed to each of the 4 loci. Table 2 lists the parameters used in the simulation.

An application of SS was evaluated in which the MOET program was reduced from 42 to 25 donors per year. The reduction in combination with using SS produced $25 \times 17=425$ female embryos per year, which was close to $42 \times 10=420$ female embryos per year in the case of RS. The comparison was then made between a smaller MOET program in which SS compensated for the smaller number of donors harvested annually and a larger system in which RS was used (comparison 7, Table 1). The objective of comparison 7 was then to evaluate the economic advantage of the smaller MOET program against its expected genetic advantage. Be- cause both the small and the large MOET programs produced almost the same number of female embryos per year, the smaller system was expected to have an economic advantage over the larger system but also to retain the same genetic level as that of the larger system. To further demonstrate that using SS was the reason behind boosting the genetic level of the smaller MOET program, a final scenario was simulated for the smaller MOET program but with RS used instead of SS.

\section{Simulation and Analysis Models}

A linear additive simulation model for milk yield was used and included management group, parity, breeding value, and residual effect. A management group was composed of herd, year, and season subclasses. The breeding value for milk yield was simulated as the aggregate effect of 40 loci in addition to 1 or 4 QTL. All loci were independent, with gametic phase equilibrium in the base population. The QTL component was simulated to study the effect of MAS in combination with SS.

The model used to analyze the simulated data was

$$
\mathbf{y}=\mathbf{X} \mathbf{b}+\mathbf{X}_{\mathrm{g}} \mathbf{g}+\mathbf{Z} \mathbf{u}+\mathbf{e}
$$

where $\mathbf{X}_{\mathrm{g}}$ is an incidence matrix relating QTL genotypes to animals with observations, and $\mathbf{g}$ is a vector of genotype effects. The matrix $\mathbf{X}$ is an incidence matrix of all other fixed factors (i.e., parity, herd, year, and season, as described in Abdel-Azim and Freeman, 2002). The vector $\mathbf{b}$ is a vector of fixed effects to be estimated. The matrix $\mathbf{Z}$ is a design matrix relating animals to their corresponding polygenic effects (u). The vector e contains random residuals specific to each individual. The total phenotypic variance used to simulate milk yield records was $(913 \mathrm{~kg})^{2}$, of which $40 \%$ was attributed to fixed effects and $60 \%$ to random effects. Heritability of the trait was assumed to be 0.3 . Details of the simulation model and its parameters can be found in AbdelAzim and Freeman (2002). 
Table 3. Genetic superiority of gender control in first-lactation cows (FLC), bull dams (BD), donor females $(\mathrm{DF})$, young bulls $(\mathrm{YB})$, and active sires $(\mathrm{AS})^{1}$

\begin{tabular}{|c|c|c|c|c|c|}
\hline Year & FLC & $\mathrm{BD}$ & DF & YB & AS \\
\hline & \multicolumn{5}{|c|}{$\longrightarrow$ (Conventional 2-stage) } \\
\hline $11-15$ & $22.4(2.3)$ & $6.0(1.4)$ & NA & $5.0(2.3)$ & $1.9(1.4)$ \\
\hline \multirow[t]{2}{*}{$16-20$} & $11.1(1.1)$ & $4.7(1.0)$ & NA & $3.1(1.4)$ & $0.4(1.1)$ \\
\hline & \multicolumn{5}{|c|}{ - (Mixed ET scheme) } \\
\hline \multicolumn{6}{|c|}{ No within-family selection of donors ${ }^{2}$} \\
\hline $11-15$ & $4.0(0.4)$ & $2.2(1.4)$ & $0.9(1.8)$ & $0.2(1.1)$ & $-0.7(0.4)$ \\
\hline \multirow[t]{2}{*}{$16-20$} & $3.5(0.5)$ & $1.9(1.1)$ & $-0.7(1.6)$ & $0.2(1.2)$ & $-0.4(1.0)$ \\
\hline & & \multicolumn{3}{|c|}{ [Mixed ET scheme with MAS (1 QTL)] } & \\
\hline \multicolumn{6}{|c|}{ No within-family selection of donors } \\
\hline $11-15$ & $1.6(0.6)$ & $2.0(1.2)$ & $1.5(1.4)$ & $0.0(1.0)$ & $0.2(0.6)$ \\
\hline $16-20$ & $1.4(0.7)$ & $1.8(0.9)$ & $0.2(1.5)$ & $0.7(1.2)$ & $0.1(0.9)$ \\
\hline \multicolumn{6}{|c|}{ Within-family selection of donors } \\
\hline $11-15$ & $0.6(0.5)$ & $1.0(1.4)$ & $-0.2(0.8)$ & $-0.1(1.0)$ & $0.5(0.7)$ \\
\hline \multirow[t]{2}{*}{$16-20$} & $0.8(0.5)$ & $2.3(1.0)$ & $1.0(0.9)$ & $-0.3(1.0)$ & $0.1(0.8)$ \\
\hline & & \multicolumn{3}{|c|}{ [Mixed ET scheme with MAS ${ }^{3}$ (4 QTL)] } & \\
\hline \multicolumn{6}{|c|}{ No within-family selection of donors } \\
\hline $11-15$ & $0.6(1.2)$ & $0.6(2.0)$ & $2.0(1.6)$ & $0.3(1.2)$ & $0.3(1.1)$ \\
\hline $16-20$ & $2.8(0.9)$ & $6.0(1.5)$ & $7.2(1.9)$ & $0.2(1.5)$ & $2.1(1.3)$ \\
\hline \multicolumn{6}{|c|}{ Within-family selection of donors } \\
\hline $11-15$ & $1.1(1.2)$ & $2.1(2.2)$ & $1.0(1.3)$ & $0.6(1.2)$ & $0.4(1.1)$ \\
\hline $16-20$ & $1.0(0.9)$ & $2.6(1.2)$ & $3.6(1.3)$ & $-0.8(1.4)$ & $1.1(1.3)$ \\
\hline
\end{tabular}

${ }^{1}$ Superiority is shown as a relative percentage to the base scheme without gender control. Standard errors are shown as parenthetical values. ET = Embryo transfer; MAS = marker-assisted selection.

${ }^{2}$ The corresponding scheme of within-family selection of donors was not simulated because embryos (donors) of the same full-sib family had the same breeding value.

${ }^{3}$ The 4 QTL collectively accounted for the same amount of genetic variance that 1 QTL accounted for.

An extension of the model of Abdel-Azim and Freeman (2002) was the addition of a juvenile hybrid nucleus scheme with a hierarchical mating design in which a centralized nucleus herd contributed $50 \%$ of the YB tested annually. The nucleus herd was started in yr 10 by harvesting ova from the best females in the population for 2 consecutive years, yr 10 and 11 . Waiting until yr 10 was essential to establish reliable EBV that provided a basis for identifying the best females. In yr 12, embryos of the females harvested in yr 10 became $1 \mathrm{yr}$ old and were flushed. This process of harvesting nucleus donors was continued until yr 20; that is, the MOET program was closed on the female side in yr 12. However, the nucleus herd continued to be open on the male side: RS and SS from the best 50\% of AS (whether originating in the nucleus herd or not) were used in embryo production. Also, YB originating in the nucleus herd were progeny tested in commercial herds just like other bulls. In this regard, the scheme was hybrid (Colleau, 1985).

\section{Evaluating the Superiority of Using SS}

For each of the scenarios studied, the simulation program was run 50 times. In the context of the current study, a run means simulating data and performing a genetic evaluation once a year for $20 \mathrm{yr}$. Results from each run constituted a replicate and included the cumulative genetic response (CGR) among other parameters. The CGR was obtained and stored each year for 5 groups: active sires (AS), YB, bull dams (BD), donor females (DF), and first-lactation cows (FLC). The CGR was calculated each year as the average of the true breeding values of living animals in each of the 5 groups.

Cumulative genetic responses were presented in terms of the percentage of superiority of animals specific to a certain group under breeding systems that used SS over corresponding animals under systems that used RS. The mean percentage of annual superiority was computed as

$$
2 \sum_{i=1}^{50}\left[\left(\overline{B V}_{S S}\right)_{i}-\left(\overline{B V}_{R S}\right)_{i}\right] /\left|\left(\overline{B V}_{R S}\right)_{i}\right|,\left|\left(\overline{B V}_{R S}\right)_{i}\right| \neq 0
$$

where $\left(\overline{B V}_{S S}\right)_{i}$ and $\left(\overline{B V}_{R S}\right)_{i}$ are the true breeding values for SS and RS schemes averaged for individuals of a specific group in the $i$ th replicate of 50. In early years, $\left(\overline{B V}_{S S}\right)_{i}$ and $\left(\overline{B V}_{R S}\right)_{i}$ may differ in sign; hence, division was by the absolute value of $\left(\overline{B V}_{R S}\right)_{i}$ in formula [2]. However, results of the first $10 \mathrm{yr}$ of the simulation were not part of the current study. 


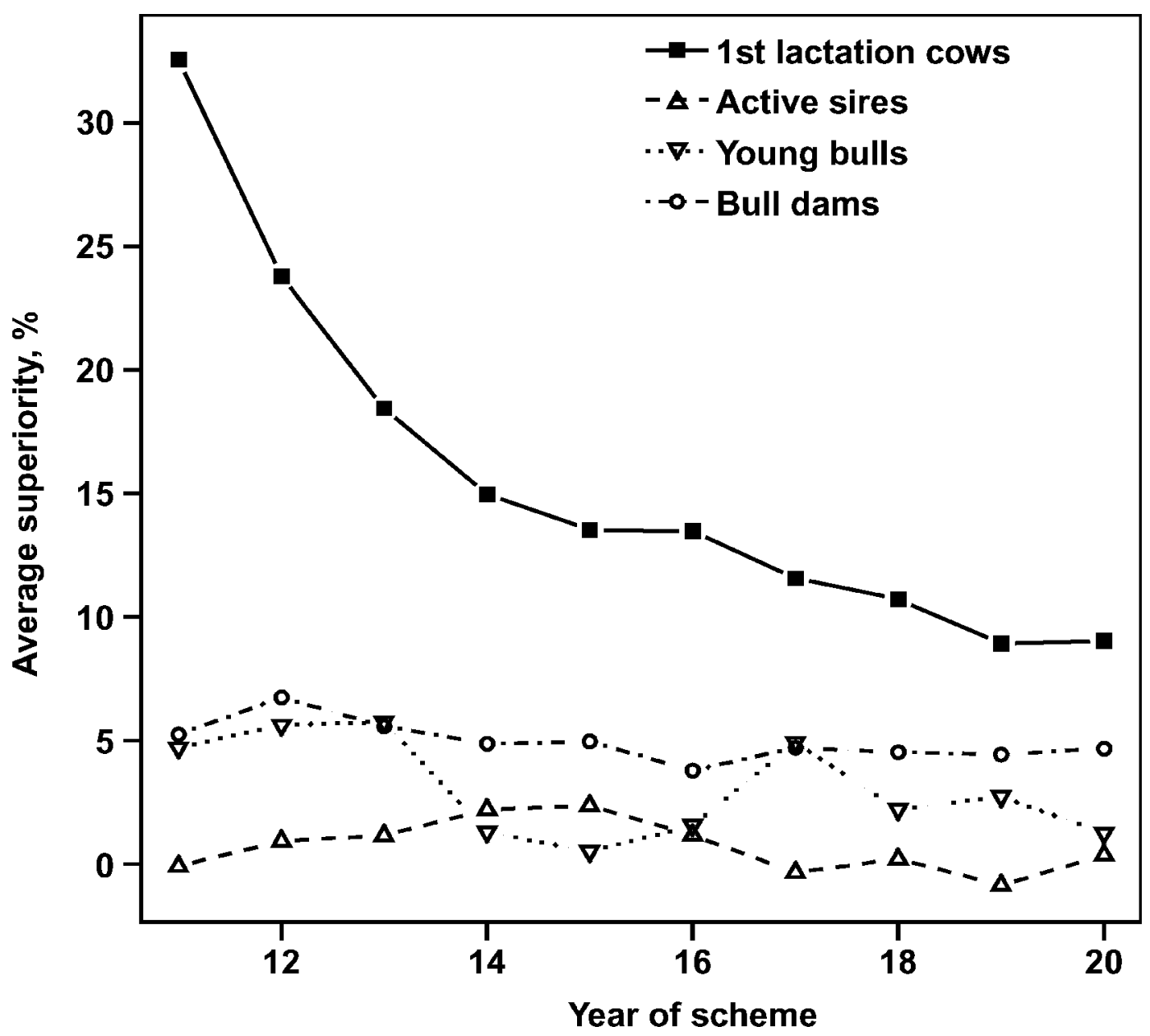

Figure 1. Impact of sorted semen on active sires, young bulls, bull dams, and first-lactation cows expressed as superiority of the sortedsemen scheme over the regular semen scheme.

\section{RESULTS AND DISCUSSION}

\section{Use of + SS in Commercial Herds}

In the scenario in which + SS was extensively used in commercial herds, a large genetic advantage was observed in commercial cows (Table 3). More females became available to herdsmen for more intense selection of herd replacements. Figure 1 illustrates the relative genetic advantage (average superiority, as calculated by formula [2]) in elite and commercial animals. The average superiority in FLC exceeded $30 \%$ in yr 11 but continued to decrease until it reached 9\% in yr 20 . The increased selection intensity in commercial cows contributed to the genetic merit of future cows (cow $\rightarrow$ cow contribution) but nucleus (elite) animals also contributed through the highly superior sires (sire $\rightarrow$ cow contribution). In fact, the contribution of elite animals grew over time and gradually marginalized the cow $\rightarrow$ cow contribution, which caused the gradual loss in average superiority observed in cows of the scheme with the higher selection intensity.

To test the significance of values in Table 3, CGR data from the scenario that used SS and the base system were merged into one data set. The data set included variables for year and gender control. Under the variable "year" were yr 6 to 20 , and under the variable "gender control" were 2 levels, SS and RS. For each group, there were a total of 1,500 observations in which each year had 100 observations, 50 from each of the 2 systems compared. By fitting the linear model [3], gender control was significant $(P<0.01)$ in all 4 groups except AS. Year was also significant in all 4 groups, which reflected the amount of genetic improvement made each year:

$$
\text { CGR }=\text { Year }+ \text { Gender Control }+\mathrm{e}
$$

In addition, annual superiority was calculated for each simulation run as 


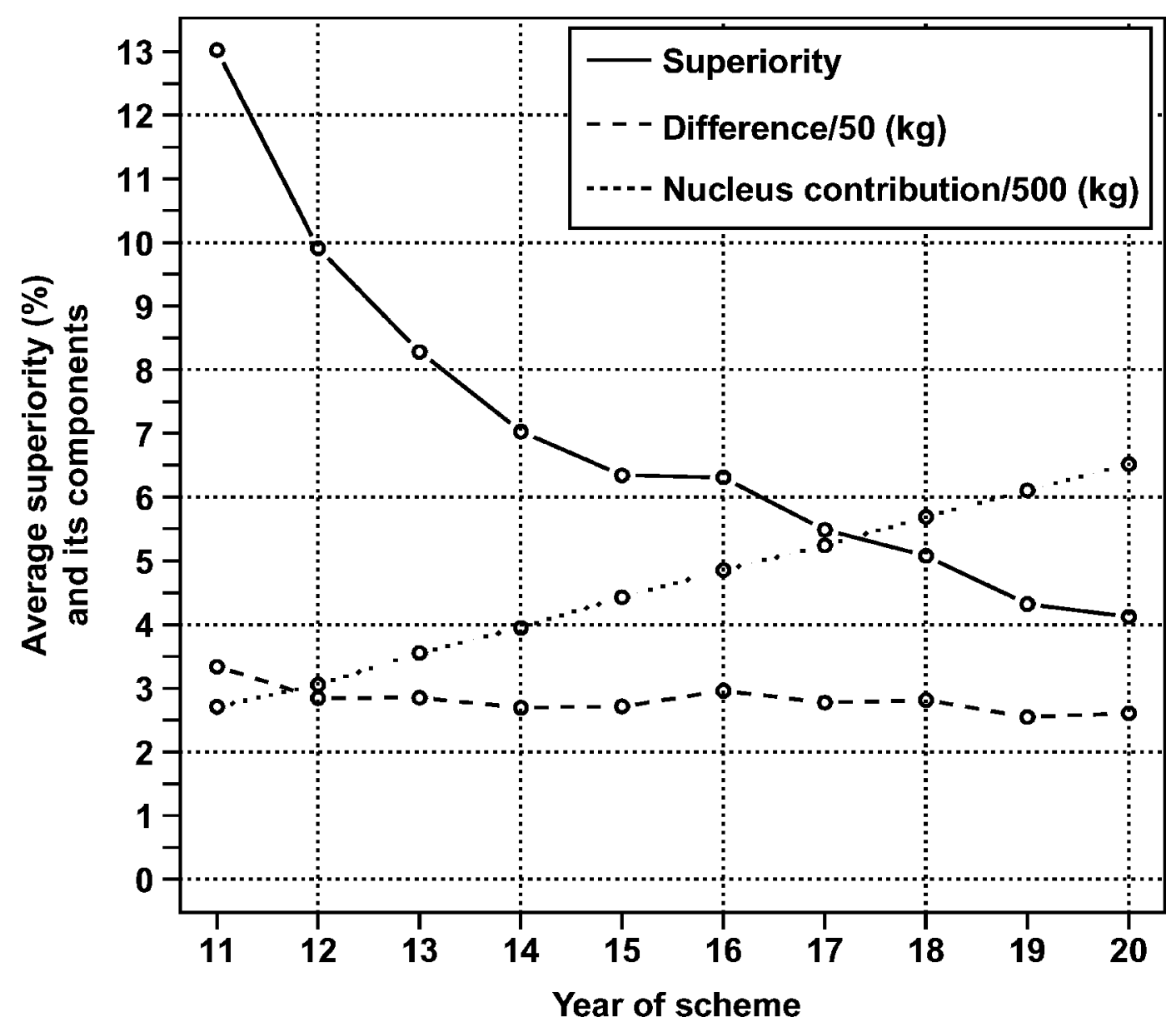

Figure 2. Superiority of first-lactation cows decomposed into components, the numerator $\left(C_{S S}-C_{R S}\right)$ divided by 50 and the denominator $\left(S_{R S}+C_{R S}\right)$ divided by 500. $S$ and $C$ are the average true breeding values of active sires and first-lactation cows in a year, and the subscripts $R S$ and $S S$ associate contributions to the regular semen and sorted semen schemes, respectively.

$$
\begin{aligned}
100\left[\left(\overline{B V}_{S S}\right)_{i}\right. & \left.-\left(\overline{B V}_{R S}\right)_{i}\right] /\left|\left(\overline{B V}_{R S}\right)_{i}\right|,\left|\left(\overline{B V}_{R S}\right)_{i}\right| \\
& \neq 0, i=1 \text { to } 50 .
\end{aligned}
$$

A regression model for superiority on yr 10 to 20 was fit for each of AS, YB, BD, and FLC separately. For each group and year, there were 50 measurements of superiority. The regression coefficient on year was found to be negative and significant $(P=0)$ only for FLC, which affirmed the gradual loss of superiority over the final $10 \mathrm{yr}$.

\section{Explaining the Gradual Loss of Superiority in FLC}

We demonstrate that the gradual superiority loss observed in FLC, as shown in Figure 1, is attributed to the growing contribution of the nucleus (AS in particular) to the $\mathrm{SS}$ and RS schemes. This loss is attributed neither to variability loss in FLC nor to intensity loss in commercial cows of the SS scheme. To show this, we explic- itly quantified the contributions of AS and FLC to future FLC, that is, the 2 selection pathways AS $\rightarrow$ FLC and FLC $\rightarrow$ FLC. If the contribution of a selection pathway is taken to be the average true breeding value of contributing animals belonging to the pathway, then the part of genetic merit of future FLC that is attributable to AS and FLC is $(S+C) / 2$, where $S$ and $C$ are average true breeding values of AS and FLC in a year. Because superiority, as expressed in formulation [2], is a relative difference, the superiority of FLC of the SS scheme is then proportional to $\left(S_{S S}+C_{S S}-S_{R S}-C_{R S}\right) /$ $\left(S_{R S}+C_{R S}\right)$, where the subscripts $R S$ and $S S$ associate contributions with the regular and SS schemes, respectively. But because we found from the current simulation that $S_{S S}$ was not significantly different from $S_{R S}$ and that the difference was generally minimal (Table 3 ), superiority could be reduced to $\left(C_{S S}-C_{R S}\right) /\left(S_{R S}+\right.$ $\left.C_{R S}\right)$. A plot of superiority with its components $\left(C_{S S}\right.$ $\left.-C_{R S}\right)$ and $\left(S_{R S}+C_{R S}\right)$ revealed that superiority loss 


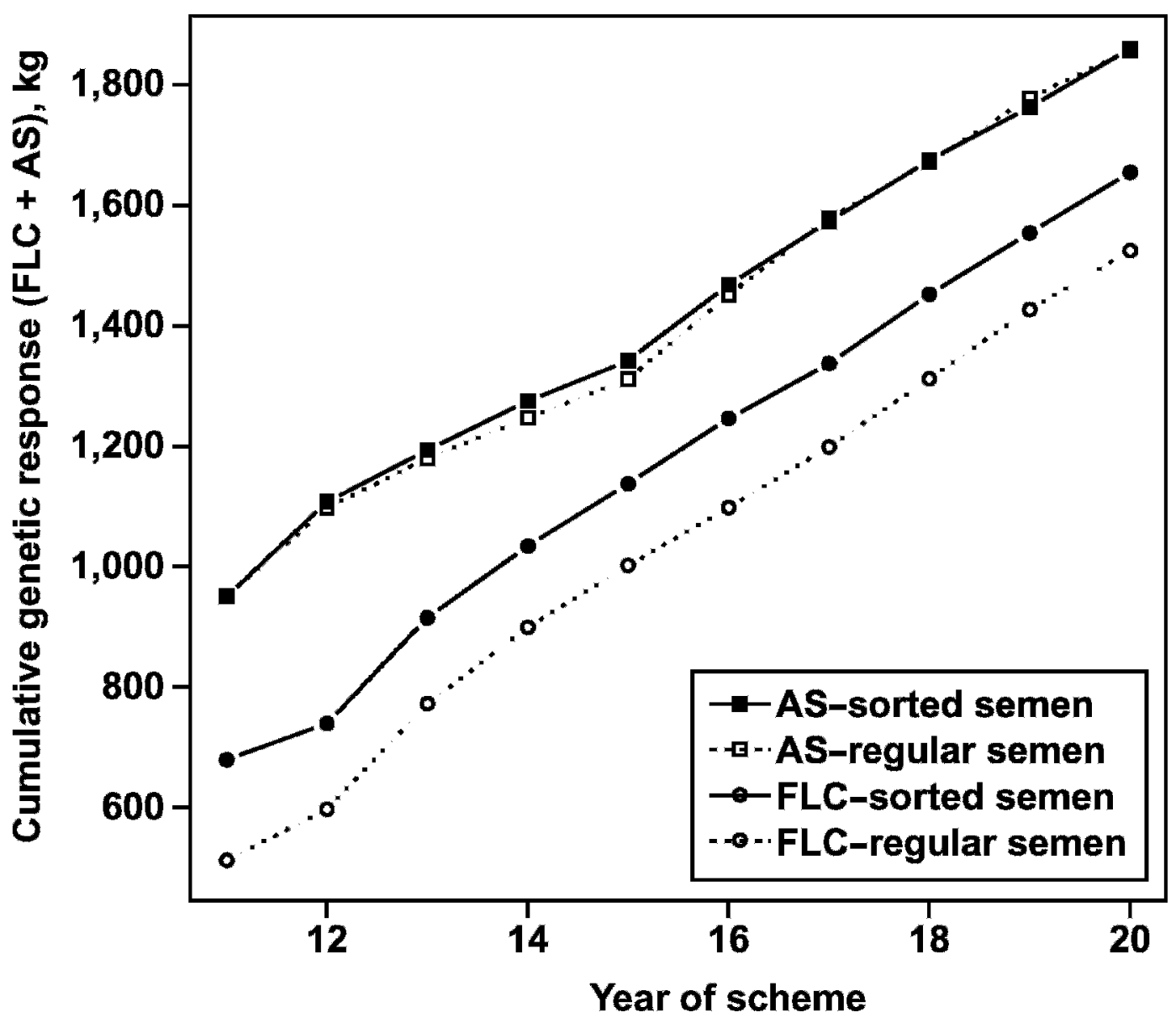

Figure 3. Cumulative genetic responses (in kg) for first-lactation cows (FLC) and active sires (AS) of 2 breeding schemes in which regular semen is used in the first and sorted semen is used in the second.

corresponded to increasing denominator and stable numerator values (Figure 2). This demonstrates that the loss observed in superiority was because of the gradual growth in nucleus contribution, which marginalized the stable difference in genetic merit between FLC of the SS and RS schemes. Figure 3 further illustrates the stable difference between FLC of the SS and RS schemes, in which the 2 lines of FLC are essentially parallel. No difference was seen between AS of the SS and RS schemes; however, the effect of genetically improving the elite individuals can be seen from the higher CGR of AS (Table 5).

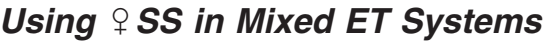

This section summarizes the simulation results of using SS in nucleus herds in which MOET took place. First, the genetic advantage of gender control in a mixed ET scheme in which i SS was used only on DF was minimal (Table 3). The positive advantage of using SS was noticed only with FLC and BD, as seen from the averages and their standard errors in Table 3. This indicates that the nucleus herd itself did not benefit from such use of SS. Females outside the nucleus herd benefited just because, in the case of SS use, more donors were released to the outside population after $1 \mathrm{yr}$ of use. Notice that increasing the number of female embryos produced in the nucleus herd as a result of using SS was not expected to have any genetic advantage. The extra number of females produced (hence, the extra selection intensity) resulted from bigger full-sib families in which females belonging to the same family were not genetically differentiable among each other at such an early age. In addition, increasing the number of female embryos by using i SS decreased the pool of males from which YB were selected, which eventually caused the slight negative disadvantage noticed with $\mathrm{AS}$.

Second, when MAS was made available (comparisons 3 to 6 , Table 1), differentiation among full-sib donors was possible. In general, MAS combined with the use of SS was effective in mixed ET schemes. Having more 
Table 4. Genetic superiority of gender control in first-lactation cows (FLC), bull dams (BD), donor females $(\mathrm{DF})$, young bulls $(\mathrm{YB})$, and active sires $(\mathrm{AS})^{1}$

\begin{tabular}{|c|c|c|c|c|c|}
\hline Year & FLC & $\mathrm{BD}$ & $\mathrm{DF}$ & YB & AS \\
\hline & - [Small & s) ET sch & † SS vs. la & lonors) ET & ith RS] \\
\hline \multirow{3}{*}{$\begin{array}{l}11-15 \\
16-20\end{array}$} & $-0.2(1.5)$ & $0.0(1.9)$ & $3.4(1.8)$ & $0.8(1.5)$ & $-0.5(1.0)$ \\
\hline & $1.4(1.0)$ & $3.7(1.3)$ & $4.9(1.6)$ & $-1.5(1.7)$ & $0.2(1.3)$ \\
\hline & [Small & s) ET sch & q SS vs. SI & donors) ET & ith RS] \\
\hline $11-15$ & $2.2(1.3)$ & $2.0(1.9)$ & $4.9(2.0)$ & $-0.4(0.9)$ & $0.6(0.9)$ \\
\hline $16-20$ & $3.8(0.9)$ & 6.9 (1.3) & $6.5(2.0)$ & $1.4(1.6)$ & $1.4(1.3)$ \\
\hline
\end{tabular}

${ }^{1}$ Superiority is shown as a relative percentage to the scheme without gender control. Advantage of a reduced multiple ovulation and embryo transfer program in which sorted semen is used is shown against 2 schemes: first, a large system in which regular semen (RS) is used, and second, a small system in which RS is used. Standard errors are shown as parenthetical values. ET = Embryo transfer; o SS = female-sorted semen.

loci in the QTL component increased diversity, and consequently increased the effectiveness of SS. With 4 loci, superiority in DF exceeded $7 \%$ and in AS exceeded $2 \%$; corresponding superiorities with one locus were 0.2 and $0.1 \%$, respectively (Table 3 ).

Within-family selection combined with MAS in mixed ET schemes might be thought to increase the effectiveness of SS relative to ignoring family structure in selection. However, simulation results showed otherwise (Table 3). A possible justification is that the increase in family size from 10 to 17 was still not sufficient to capture all the genotypic diversity in the case of the QTL component with 4 loci. On the other hand, the benefit of increasing the family size in the case of the QTL component with one locus was limited because only 3 different genotypes existed.

The absolute CGR is smaller with 4 than 1 QTL (Table 5 ) because when the number of loci increases within the same amount of additive genetic variation, their effects become smaller; hence, the selection pressure on them becomes smaller (Falconer, 1989), which results in less genetic response per unit time.

\section{Reducing the MOET Program}

Results of the reduced MOET program are presented in Table 4. First, using SS in a smaller MOET program was not associated with any major genetic disadvantage. In fact, BD and DF benefited from the use of SS (+3.7 and $+4.9 \%$, respectively), even after reducing the number of donors harvested annually (Table 4). To further demonstrate the advantage of the smaller system when combined with the use of $q$ SS, its advantage was calculated relative to the same system when combined with the use of RS. As conceptually expected, the advantage relative to the small system when combined with

Table 5. Cumulative genetic response $(\mathrm{CGR}, \mathrm{kg})^{1}$ of all base (without gender control) schemes studied ${ }^{2}$

\begin{tabular}{lrrrrrr}
\hline Breeding system & Year & FLC & BD & DF & YB & AS \\
\hline Conventional 2-stage & $11-15$ & 757 & 1,280 & NA & 867 & 1,157 \\
& $16-20$ & 1,312 & 1,771 & NA & 1,391 & 1,667 \\
Mixed ET 3 scheme & $11-15$ & 807 & 1,387 & 1,340 & 849 & 1,156 \\
& $16-20$ & 1,408 & 1,938 & 1,822 & 1,438 & 1,677 \\
Mixed ET 3 scheme with MAS (1 QTL) & $11-15$ & 1,050 & 1,465 & 1,470 & 891 & 1,149 \\
No WFS of donors & $16-20$ & 1,446 & 1,960 & 1,852 & 1,511 & 1,724 \\
WFS of donors & $11-15$ & 1,025 & 1,479 & 1,259 & 892 & 1,149 \\
& $16-20$ & 1,391 & 1,911 & 1,607 & 1,490 & 1,701 \\
Mixed ET 3 scheme with MAS (4 QTL) & $11-15$ & 846 & 1,141 & 1,148 & 713 & 915 \\
No WFS of donors & $16-20$ & 1,168 & 1,627 & 1,562 & 1,209 & 1,375 \\
WFS of donors & $11-15$ & 821 & 1,129 & 997 & 714 & 913 \\
& $16-20$ & 1,134 & 1,624 & 1,406 & 1,184 & 1,359 \\
Small ${ }^{4}$ mixed ET scheme & $11-15$ & 826 & 1,115 & 1,131 & 717 & 907 \\
& $16-20$ & 1,138 & 1,580 & 1,540 & 1,172 & 1,355 \\
\hline
\end{tabular}

${ }^{1}$ Additive genetic variance $=\mathrm{h}^{2}(0.6)(913 \mathrm{~kg})^{2} . \mathrm{ET}=$ embryo transfer; MAS = marker-assisted selection; WFS = within-family selection; i $\mathrm{SS}$ = female-sorted semen; $\mathrm{RS}=$ regular semen.

${ }^{2}$ Base response is shown for first-lactation cows (FLC), bull dams (BD), donor females (DF), young bulls (YB), and active sires (AS).

${ }^{3} 42$ donors selected each year from $420(\mathrm{RS})$ and 714 ( $\left.9 \mathrm{SS}\right)$.

${ }^{4} 25$ donors selected each year from $250(\mathrm{RS})$ and $425(\uparrow \mathrm{SS})$ 
Table 6. Inbreeding coefficients for the breeding systems studied, calculated as an average of all animals in the analysis in yr 10 and 20 for both female-sorted semen ( $+\mathrm{SS})$ and regular semen $(\mathrm{RS})^{1}$

\begin{tabular}{|c|c|c|c|c|}
\hline \multirow[b]{2}{*}{ Breeding system } & \multicolumn{2}{|c|}{ is } & \multicolumn{2}{|c|}{$\mathrm{RS}$} \\
\hline & Yr 10 & Yr 20 & Yr 10 & Yr 20 \\
\hline Conventional 2-stage & 0.0199 & 0.0619 & 0.0152 & 0.0420 \\
\hline Mixed $\mathrm{ET}^{2}$ scheme & 0.0152 & 0.0482 & 0.0152 & 0.0521 \\
\hline \multicolumn{5}{|c|}{ Mixed $\mathrm{ET}^{2}$ scheme with MAS (1 QTL) } \\
\hline No WFS of donors & 0.0099 & 0.0731 & 0.0099 & 0.0482 \\
\hline WFS of donors & 0.0099 & 0.0576 & 0.0099 & 0.0415 \\
\hline \multicolumn{5}{|c|}{ Mixed $\mathrm{ET}^{2}$ scheme with MAS (4 QTL) } \\
\hline No WFS of donors & 0.0091 & 0.0710 & 0.0091 & 0.0497 \\
\hline WFS of donors & 0.0091 & 0.0642 & 0.0091 & 0.0471 \\
\hline Small $^{3}$ mixed ET scheme & 0.0091 & 0.0504 & 0.0091 & 0.0387 \\
\hline
\end{tabular}

${ }^{1} \mathrm{ET}=$ embryo transfer; MAS = marker-assisted selection; WFS = within-family selection.

${ }^{2} 42$ donors selected each year from 420 (RS) and 714 ( $\%$ SS).

${ }^{3} 25$ donors selected each year from 250 (RS) and 425 ( $+\mathrm{SS}$ ).

RS was found to be much larger than the advantage relative to the large system when combined with $\mathrm{RS}$ (Table 4). The results presented justify establishing smaller MOET programs if i SS is used to increase the pool of female embryos, even with a corresponding reduction in male embryos.

\section{Rate of Inbreeding}

Because simulation was stochastic, inbreeding coefficients were averaged each year for all animals in the pedigree file. Table 6 lists inbreeding coefficients of yr 10 and 20. The pedigree file included all animals that contributed to the analyses performed in yr 6 to 20; no pedigree reduction process was implemented before any of the annual analyses.

In general, SS schemes had slightly higher (around +0.02 after $20 \mathrm{yr}$ of simulation) inbreeding coefficients, but no drastic differences were noticed between RS and SS schemes in any of the breeding systems studied. Also, breeding systems that used WFS resulted in a minor reduction of the inbreeding rate, but that was accompanied by a corresponding reduction in CGR (Table 5). The reduction in the inbreeding rate was more pronounced with + SS (Table 6).

\section{CONCLUSIONS}

This study quantified the effect of $q$ SS on the genetic progress of elite and commercial animals in a dairy cattle population. In a 2-stage selection scheme with SS widely available to commercial herds, we found that the impact on commercial cows was large. Although using + SS outside the nucleus increased the intensity of selection among commercial cows and boosted genetic progress, the nucleus contribution grew over time and marginalized the cow-to-cow contribution.
Second, the effects of SS inside the nucleus were evaluated: A mixed juvenile multiple ovulation and ET scheme was evaluated by simulating a centralized nucleus herd that partially contributed to the YB tested annually. Sorted semen did not change genetic progress in juvenile schemes. Only when MAS was implemented in combination with SS was more genetic progress made relative to MAS alone.

Two other factors that influenced the effectiveness of MAS when combined with SS were studied: WFS of DF and the number of loci in the QTL component. First, WFS was not found to be superior to the selection of donors regardless of their familial structure, and the number of loci was found to be an important factor in increasing the effectiveness of MAS in combination with SS. Further, a certain application was studied to reduce the nucleus size by harvesting fewer donors but producing the same number of female embryos by using SS. Using SS in a reduced-nucleus scheme gained genetic progress that was at least as good as a large-nucleus scheme with RS.

Finally, 2 important considerations need to be emphasized with regard to the current study. First, ET schemes contributed half, not all, of the YB tested annually, with the other half being unaffected by SS. Second, SS was used only on DF, not on any other females in the population. If SS is implemented in practice, it will be used more extensively both inside and outside the nucleus, which will have greater effects on genetic progress in dairy cattle populations.

\section{REFERENCES}

Abdel-Azim, G., and A. E. Freeman. 2002. Superiority of QTL-assisted selection in dairy cattle breeding schemes. J. Dairy Sci. 85:1869-1880. 
Baker, R. L., P. Shannon, D. J. Garrick, H. T. Blair, and B. W. Wickham. 1990. The future impact of new opportunities in reproductive physiology and molecular biology on genetic improvement programmes. Proc. N. Z. Soc. Anim. Prod. 50:197-210.

Colleau, J. J. 1985. Genetic improvement by embryo transfer within selection nuclei in dairy cattle. Genet. Sel. Evol. 17:499-538.

Colleau, J. J. 1991. Using embryo sexing within closed mixed multiple ovulation and embryo transfer schemes for selection in dairy cattle. J. Dairy Sci. 74:3973-3984.

Falconer, D. S. 1989. Introduction to Quantitative Genetics. 3rd ed. Longman, New York, NY.
Kinghorn, B. P., C. Smith, and J. C. M. Dekkers. 1991. Potential genetic gains in dairy cattle with gamete harvesting and in vitro fertilization. J. Dairy Sci. 74:611-622.

Lush, J. L. 1945. Animal Breeding Plans. The Iowa State College Press, Ames, IA

Van Vleck, L. D. 1981. Potential genetic impact of artificial insemination, sex selection, embryo transfer, cloning and selfing in dairy cattle. Pages 222-242 in New Technologies in Animal Breeding. B. G. Brackett, G. E. Seidel, and S. M. Seidel, ed. Academic Press, New York, NY.

Weigel, K. A. 2004. Exploring the role of sexed semen in dairy production systems. J. Dairy Sci. 87(E Suppl.):E120-E130. 